\title{
7. DRAMATISCHE ALLEGORIEN
}

Trotz der Unterschiede zwichen den größeren Dialogtypen - nämlich Lehrdialogen, Streitgesprächen, philosophischen und selbstbetrachtenden Dialogen -, lassen sich einige übergreifende, allen gemeinsame Eigenschaften beobachten: die schwache Entwicklung fiktiver Situationen, die mangelnde Charakterisierung der Figuren, die Abwesenheit einer Handlung und die Vorliebe für Zwiegespräche. Allerdings finden sich auch immer wieder Dialoge, die einige dieser Merkmale nicht aufweisen und dadurch eine Sonderstellung innerhalb ihrer Gruppe einnehmen, wie zum Beispiel die Religionsgespräche des Raimundus Lullus aus der ersten Zeit seines Schaffens ${ }^{1}$. In diesem Kapitel werden nun einige Texte behandelt, die diese übergreifenden Merkmale geradezu ins Gegenteil verkehren: Sie entwickeln nämlich eine fiktive Handlung, die im Gespräch vorangetrieben wird und an der sich mehrere Figuren beteiligen. Es handelt sich um folgende Werke: der Liber natalis pueri parvuli Christi Jesu (R32n), der Liber lamentationis Philosophiae (R32o) und der Liber de civitate mundi (R32x) von Raimundus Lullus, der Liber dialogorum Johanns von Jenstein (R67a) und die Legatio prophetarum ad Deum pro incarnatione Filii Dei (R77), die in einer Handschrift dem ansonsten unbekannten Frater Nicolaus de Spina zugeschrieben wird.

Die Gespräche sind in diesen Texten in eine allegorische Fiktion eingebettet. Lullus will dadurch die Lage des zeitgenössischen Christentums und die ihm drohenden Gefahren darstellen, um einen Weg aus dieser Situation zu weisen. Die Legatio prophetarum stellt die Heilsgeschichte vom Sündenfall des Menschen bis zu seiner Erlösung dar, während Johann von Jenstein das Heilsgeschehen am Beispiel eines einzelnen Menschen vorführt. Die auftretenden Personen sind allegorische Figuren (zum Beispiel die Tugenden im Liber natalis und die Eigenschaften Gottes Dignitates - im Liber de civitate mundi), Personifizierungen (Körper und Seele bei Johann von Jenstein) oder Akteure des Heilsgeschehens, entweder als Individuen (Jesus, Maria, die Propheten in der Legatio prophetarum) oder als Vertreter einer Gruppe (Engel, Teufel, Mensch im Liber dialogorum). Die Figuren stehen in unterschiedlicher Beziehung

\footnotetext{
${ }^{1}$ Siehe oben $123-126$.
} 
zueinander: Sie können als Gruppe auftreten, miteinander kooperieren oder sich gegenseitig in einem Vorhaben behindern. Die Personensprache erfüllt mehrere Funktionen: Durch sie entwickelt sich die Handlung, die Figuren werden charakterisiert und ihre gegenseitige Beziehung vorgeführt. Innerhalb der Werke finden sich verschiedene Gesprächsformen: Beratungsgespräche, in denen eine Gruppe ein gemeinsames Vorgehen entscheidet (der Rat der Dignitates im Liber de civitate mundi, der Rat der Dreieinigkeit und die Versammlung der Gerechten in der Vorhölle, beide in der Legatio prophetarum), Streitgespräche, in denen verschiedene Positionen pointiert vertreten werden - wie im Liber dialogorum zwischen Engel und Teufel sowie zwischen Körper und Seele - und Lehrgespräche (zum Beispiel, zwischen Engel und Mensch über die Jenseitswelt, auch im Liber dialogorum). Eine besondere Form der Figurendarstellung sind die Reden, mit denen die allegorischen Gestalten sich in den drei Werken des Lullus vorstellen. Obwohl die Handlung durch die Dialoge vorangetrieben wird, schaltet sich gelegentlich ein Erzähler ein. Am häufigsten geschieht dies in der Legatio prophetarum, in der die Dialogabschnitte durch erzählende Passagen verbunden sind, so dass der Aufbau des Textes sich der Romanstruktur annähert.

Die dramatischen Allegorien sind inhaltlich verschiedenen Texttraditionen verpflichtet, denn allegorische Erzählungen stellen in der mittellateinischen Literatur wegen ihres didaktischen Wertes eine geschätzte Form der literarischen Fiktion dar. Das allegorische Epos entstand im 4. Jahrhundert mit der Psychomachia des Prudentius, deren Verbreitung und Einfluss im Mittelalter kaum zu überschätzen sind. Im Hochmittelalter entstanden weitere einflussreiche allegorische Epen mit philosophischem Inhalt, wie der Anticlaudianus des Alanus ab Insulis und die Cosmographia des Bernardus Silvestris, sowie allegorische Prosimetra, die den Einfluss von Boethius und Martianus Capella erkennen lassen². In beiden Textsorten sind Szenen nach dem Vorbild des Martianus anzutreffen, in denen die Artes sich selbst und ihre jeweiligen Inhalte vorstellen ${ }^{3}$. Diese Art der Selbstdarstellung könnte die Reden der Figuren in den dramatischen Allegorien des Lullus beeinflusst haben.

Kleine allegorische Szenen werden außerdem in längere Texte wie Predigten oder Bibelkommentare zur Verdeutlichung einer theoretischen

${ }^{2} \mathrm{Zu}$ den allegorischen-philosophischen Prosimetra siehe oben 172-174.

${ }^{3}$ Zum Beispiel in De eodem et diverso Adelards von Bath (siehe oben 173) und in De consolatione rationis des Petrus Compostellanus (R43, siehe oben 200-201). 
Diskussion eingegliedert. Ohly hat in seiner Untersuchung dreier solcher Szenen - der Schöpfungsrat und der Erlösungsrat der Trinität, der Streit der Töchter Gottes - ihre Wandelbarkeit und Verbreitung gezeigt:

Wie bei solchen Mythen üblich, stimulierte die an keine biblische Tradition gebundene Szene des Erlösungsrats innerhalb der Trinität oder der vier Tugenden oder beider zusammen die Geister zu den verschiedensten Ansätzen der Entfaltung und zu freien Abwandlungen des Motivs, die in frischer Farbigkeit nebeneinanderstehen, so daß von einem guten Dutzend Textzeugen des 12. Jahrhunderts alle Eigenständigkeit offenbaren ${ }^{4}, \ldots$

Ohlys Darstellung zeigt eindrücklich die Schwierigkeit, bei solch weit verbreiteten allegorischen Szenen bereits für Texte des 12. Jahrhunderts inhaltliche Abhängigkeiten zu bestimmen. Im Spätmittelalter wäre die Quellenidentifizierung ein unmögliches Unterfangen. In Liber dialogorum Johanns von Jenstein und in der Legatio prophetarum sind Motive zu finden, die auch im theologischen Fachschrifttum, in Dramen, in Exempeln und in Meditationen zum Leben Jesu sowohl in Latein als auch in den Volkssprachen in ähnlicher Form anzutreffen sind: der Rat der Trinität, der Streit der Töchter Gottes, der Streit von Körper und Seele, der Verkauf der Seele an den Teufel, der Kampf von Engeln und Teufeln um die Seele ${ }^{5}$. Johann von Jenstein, der seinen Liber als Abfolge von Zwiegesprächen strukturiert, lässt in den einzelnen Gesprächen Ähnlichkeiten mit Dialogtraditionen erkennen: Der Streit zwischen Engel und Teufel zeigt Parallelen zu den Streitgesprächen über das Heilsgeschehen, während die Belehrung des Menschen durch den Engel über das Jenseits an die Soliloquien Hugos von St. Victor und Bonaventuras erinnert. Der Streit zwischen Körper und Seele, die sich gegenseitig für die Sünde und die zu erleidende Strafe verantwortlich machen, ist in Streitgedichten anzutreffen, vor allem in der bekannten Visio Philiberti ${ }^{6}$. Das Thema wird auch in einem Prosimetrum des 12. Jahrhunderts behandelt, im Liber de Querimonia Hildeberts de Lavardin?

${ }^{4}$ Ohly, „Die Trinität“, 258-259.

$\mathrm{Zu}$ den zwei letzten Themen vgl. Walther, Das Streitgedicht, 84-85.

Vgl. Walther, Das Streitgedicht, 63-74.

${ }^{7}$ Die Visio Philiberti oder Altercatio corporis et animae ist in der ersten Hälfte des 13. Jhs., wohl in England, entstanden. Sie war sehr weit verbreitet und wurde in mehrere Volkssprachen übersetzt, vgl. Palmer, „Visio“. Es gibt keine Einigkeit in der Forschung über eine mögliche Abhängigkeit der Visio Philiberti vom Liber de querimonia, vgl. Hildebert, Liber de querimonia, Ed. Orth, 56, Anm. 128 und oben 172-173. Der Liber dialogorum ist noch unediert und unerschlossen. 
Die allegorischen Szenen, die in längere Texte integriert sind, verwenden zum Teil die Personensprache als Darstellungsform. Als Anregung für die Form der dramatischen Allegorien dürften aber vornehmlich selbstständige Texte in Frage kommen. Hier sind allegorische Gedichte zu erwähnen, die den Streit der Töchter Gottes oder der Kampf der Tugenden und Laster in Personensprache darstellen. Walther und Schmidt betrachten sie als Streitgedichte, doch bilden sie wegen der Entwicklung einer Handlung mit mehreren Personen eine besondere Gruppe, darin den dramatischen Allegorien nicht unähnlich ${ }^{8}$. Parallelen zu den dramatischen Allegorien finden sich auch in der Textsorte der Parabeln, kurze Texte, die mit der Hilfe von Gleichnissen schwierige theologische Inhalte in einfachen Worten vermitteln ${ }^{9}$. Sie dürften ihren Ursprung in mündlichen Formen der klösterlichen Unterweisung haben: in der Predigt, der Collatio oder auch in der zwanglosen Unterhaltung innerhalb des Klosters. Dies kann für die Parabeln Anselms von Canterbury nachgewiesen werden, die von seinen zwei Sekretären, Alexander und Eadmer, gesammelt und herausgegeben wurden ${ }^{10}$. Auch bei den Zisterziensern entstanden Parabelsammlungen, im 12. Jahrhundert sind zu nennen die Parabolae Bernhards von Clairvaux ${ }^{11}$ und das gleichnamige Werk Galands von Reigny. Ende des 12. Jahrhunderts leitet das Similitudinarium des Lehrers in der Kathedralschule von Lincoln, William de Montibus, eine Loslösung der Form von der monastischen Literatur und folglich eine Erweiterung des Publikums ein ${ }^{12}$. William verfasste mehrere Werke, die den Klerus in seiner Pastoraltätigkeit unterstützen sollten, darunter Sammlungen von Argumenten und Zitaten als Predigerhilfen. Auch das Similitudinarium bietet Texte, die als illustrierende

${ }^{8}$ Vgl. Walther, Das Streitgedicht, 110-126 und P. G. Schmidt, „I conflictus“, 158-164. Letzerer erwähnt in diesem Zusammenhang ein anonymes Gedicht zum Streit der Töchter Gottes, den Conflictus virtutum et vitiorum des Willelm Jordaens und das Certamen anime des Raymundus de Rocosello.

${ }^{9} \mathrm{Zu}$ dieser Textsorte vgl. Leclercq, „Introduction“, Galand de Reigny, Parabolarium, Ed. Friedlander / Leclercq / Raciti, 22-30. Allerdings fasst Leclercq den Begriff „Parabel“ sehr weit, so dass er alle lehrhafte allegorische Darstellungen umfasst.

${ }^{10}$ In folgenden Werken: De humanis moribus und Liber ex dictis beati Anselmi von Alexander sowie Eadmers De beatitudine perennis vitae. Die drei Texte wurden von Southern / Schmitt ediert. Später entstand eine Sammlung mit dem Titel Liber de similitudinibus, die Material aus De humanis moribus, De beatitudine perennis vitae und Eadmers Vita Ansemi vereinigt. Vgl. Southern, Saint Anselm, 382-394 und die Einführung zu Southern / Schmitt, 4-35.

${ }^{11}$ Ediert in Opera omnia, Ed. Leclercq, Bd. 6, 2, 257-303.

12 Über William de Montibus vgl. Goering, William, über das Similitudinarium ebd. 304-312. 
Beispiele in einer Predigt eingesetzt werden können: Parabeln, historische Erzählungen und Vergleiche aus der Natur ${ }^{13}$. Anselms Parabeln wurden im Spätmittelalter häufig wegen der darin erläuterten Inhalte in den Universitäten gelesen und kommentiert ${ }^{14}$. In den Parabelsammlungen sind ähnliche Themen wie in den dramatischen Allegorien anzutreffen: Bei Galand von Reigny findet sich zum Beispiel eine kurze Darstellung der Heilsgeschichte ${ }^{15}$, die an die viel ausführlichere in der Legatio prophetarum erinnert, während die erste Parabel Bernhards von Clairvaux eine allegorische Darstellung von der Suche der Seele nach ihrem Heil bietet, die eine gewisse Ähnlichkeit mit dem Liber dialogorum aufweist ${ }^{16}$. Einzelne Parabeln konnten auch selbstständig zirkulieren. Ein Beispiel dafür ist De custodia interioris hominis, das im Mittelalter Anselm zugeschrieben wurde ${ }^{17}$. Der Text weist eine ähnliche Gestaltung wie die dramatischen Allegorien auf: Nach einer kurzen Einleitung, in der der Verfasser auf Matth 24,43 hinweist, wird in einer dialogischen Szene vorgeführt, wie die Tugenden die Seele vor ihrem Feind retten. Prudentia tritt als Hausherr der menschlichen Seele auf und berät sich mit Memoria mortis, den vier Kardinaltugenden und dem Desiderium vitae aeternae.

Die dramatischen Allegorien wirken wie stark erweiterte dialogisierte Parabeln, allerdings dürfte ein weiterer Einfluss eine Rolle gespielt haben: das Drama, in Latein und hauptsächlich in den Volkssprachen. In den geistlichen Dramen des Spätmittelalters sind ähnliche Themen und Szenen anzutreffen und die charakteristischen Merkmale der dramatischen Allegorien erinnern an die literarischen Gestaltungsmittel des Theaters: die Charakterisierung der Personen durch ihre Sprache, die Entwicklung einer Handlung, die unterschiedlichen, sich verändernden

${ }^{13}$ William war um die Benutzerfreundlichkeit seiner Sammlung bemüht und organisierte deshalb die Texte nach Stichworten in alphabetischer Reihenfolge. In der Praefatio erwähnt er ausdrücklich die Verwendbarkeit in Predigten: Ad declarandum in sermone quocumque propositum similitudines undecumque Deo donante collegimus, scientes quia propositiones in medium prolatas probant seu dilucidant auctoritates et rationes, exempla et similitudines (Goering, William, 313). Die Praefatio und Exzerpte wurden von Goering, William, 313-333, ediert.

${ }_{14}$ Vgl. Southern, Saint Anselm, 390-391.

${ }^{15}$ Ed. Friedlander / Leclercq / Raciti, 56-63.

${ }_{16}$ „De filio regis“, Opera omnia, Ed. Leclercq, 6, 2, 261-266.

17 Die Frage der Autorschaft ist ungeklärt, die ältesten Textzeugen sind Mitte des 12. Jahrhunderts entstanden. Der Text ist ediert bei Southern / Schmitt, 354-360. Für die Autorschaft Anselms sprach sich Southern aus, „St. Anselm and his English pupils“, 11-12. 
Beziehungen der Figuren. Vollmann hat beobachtet, dass das Theater im Spätmittelalter eine Entwicklung nimmt, die mit ihrer Suche nach Spektakel und effektvollen Auftritten das Drama von der klerikalen Kultur entfernt ${ }^{18}$. Die dramatischen Allegorien nehmen die literarischen Gestaltungsmöglichkeiten des Theaters auf und kombinieren sie mit einer ernsthafteren, wenn auch nicht tiefgründigen Behandlung theologischer Themen. Sie sind Lesedramen, die sich von allzu populären oder groben Bestandteilen fernhalten und so den Reiz der dramatischen Form für ein gebildetes Publikum annehmbar machen.

Letzeres ist eine Vermutung, die durch eine Untersuchung der Rezeption zu überprüfen wäre. Was sich bisher in diesem Zusammenhang beobachten lässt, widerspricht ihr nicht. Johann von Jenstein widmete seinen Liber Johann von Neumarkt und schickte eine Abschrift an den Papst. Die unmittelbaren Empfänger sind gebildete Kleriker, die die gewählte Sprache Johanns verstehen und goutieren konnten. Lullus verfasste diese Werke, wie viele andere auch, um hochgestellte Persönlichkeiten zu einem bestimmten Vorgehen zu bewegen. Der Liber natalis und der Liber lamentationis sind an den französischen König adressiert, der Liber de civitate mundi richtete sich an die Kurie und die christlichen Herrscher. In den drei Fällen dürfte er diese an das Theater erinnernde Form gewählt haben, um besonders die adligen Empfänger, die Könige und ihre Höfe, anzusprechen. Die Legatio prophetarum ist lediglich in zwei Handschriften überliefert, in der gedruckten Fassung mit dem Titel Disputatio Trinitatis dürfte sie jedoch ein breites Publikum erreicht haben, das auch einfache Kleriker und gebildete Laien miteinschloss.

Die fünf dramatischen Allegorien lassen keine Gruppenbildung erkennen, die Verfasser scheinen nicht voneinander gewusst zu haben und folgten auch nicht denselben Vorbildern. Die Gemeinsamkeiten durch die verwendeten Motive dürften auf deren Allgegenwart im Spätmittelalter zurückzuführen sein.

${ }^{18}$ Vollmann, „Lateinisches Schauspiel des Spätmittelalters?“. 